\title{
Opioids in COPD: a cause of death or a marker of illness severity?
}

\author{
To the Editor:
}

In a recent issue of the European Respiratory Journal, Vozoris et al. [1] reported a study showing an association between opioid use and increased mortality in patients with chronic obstructive pulmonary disease (COPD). The authors concluded that these results were "worrisome", and cautioned readers about the "potential for adverse respiratory outcomes" when using opioids in patients with COPD. We are concerned about the authors' interpretation of their data, and the implication that the use of opioids is dangerous in this population.

Observational studies show that opioid use is associated with increased adjusted mortality by any cause in a variety of populations [2], but studies also show that the use of symptom-control medications such as opioids increases with age and proximity to death [3]. This suggests that opioids are probably a marker of advancing illness rather than simply a cause of death [2, 3]. Екsтrom et al. [4] also found an association between higher dose opioids and increased mortality in oxygen-dependent COPD patients; however, retrospective population-based studies will always be limited by the inability to capture the indication for opioid use, and match controls for disease severity of treated individuals. Since refractory dyspnoea is a typical indication for opioid use, and dyspnoea is also well-correlated with mortality in COPD, then a failure to adjust the analysis for dyspnoea severity in the studies by Екsтroм et al. [4] and Vozoris et al. [1] carries a substantial risk of confounding by indication [5]. In both studies, we cannot exclude the possibility that opioid use was simply a marker of presence of refractory dyspnoea and a sign of disease severity.

Of course, observational studies cannot prove that opioids are safe either. But even if we speculate that opioids are partially to blame for the observed mortality risk in the study by Vozonis et al. [1], we would have trouble explaining why low-dose incident opioid use is apparently associated with a higher risk of mortality than high-dose incident opioid use, both on a relative and absolute basis. If opioids are truly harmful, then why would this effect be mitigated by more opioids?

Ultimately, the risk and benefit of any therapy is better assessed by prospective trials than studies based solely on retrospective administrative database analysis. The authors acknowledge some of the many clinical trials that have demonstrated the safety, efficacy and effectiveness of opioids for treating dyspnoea in patients with COPD [6-8]. It is possible that these studies failed to detect harm because they lacked sufficient power, but it would have taken a very large study to detect the small mortality difference shown by Vozoris et al. [1] ( $0.8 \%$ which corresponds to a number needed to harm of $\sim 125)$. These prospective studies were instead powered to detect the benefit of opioids for the palliation of breathlessness, for which the number needed to treat is as low as 1.1 [7] to 1.6 [6].

Vozoris et al. [1] also excluded patients who had been seen by a palliative care physician, which may have eliminated many patients for whom opioids would be most appropriate. Currently, opioids are the only pharmacological therapy recommended by the Canadian Thoracic Society guidelines for the management of refractory dyspnoea in advanced COPD [9]. We wish to caution the authors and readers not to make broad conclusions about the risks of opioids in this population based on retrospective data mining, when prospective trial data does not show these harms and suggests such clear and meaningful patient-related benefits.

The stigma around opioid use leads to their underuse and contributes to many patients with advanced COPD suffering unnecessary, prolonged and severe dyspnoea as they progress towards the end of life. Fortunately, through appropriate use of symptom-targeted opioids using an individualised approach as the authors suggest, this "adverse respiratory outcome" can be safely treated [9].

Opioid use associated with mortality in COPD, but dyspnoea-targeted opioids are safe and effective (and good practice) http://ow.ly/EHiu302ST5O

James Downar ${ }^{1}$, Rebecca Colman ${ }^{1}$, Robert Horton ${ }^{2}$, Paul Hernandez ${ }^{3}$ and Graeme Rocker ${ }^{4}$

${ }^{1}$ Dept of Medicine, University of Toronto, Toronto, ON, Canada. ${ }^{2}$ Division of Palliative Medicine, Dalhousie University, Halifax, NS, Canada. ${ }^{3}$ Dept of Medicine, Dalhousie University, Halifax, NS, Canada. ${ }^{4}$ Division of Respirology, Dalhousie University, Halifax, NS, Canada. 
Correspondence: James Downar, Dept of Medicine, University of Toronto, 200 Elizabeth St. 9N-926, Toronto, ON, Canada. E-mail: james.downar@utoronto.ca

Received: July 192016 | Accepted: July 222016

Conflict of interest: Disclosures can be found alongside this article at erj.ersjournals.com

\section{References}

1 Vozoris NT, Wang X, Fischer HD, et al. Incident opioid drug use and adverse respiratory outcomes among older adults with COPD. Eur Respir J 2016; 48: 683-693.

2 Ray WA, Chung CP, Murray KT, et al. Prescription of long-acting opioids and mortality in patients with chronic noncancer pain. JAMA 2016; 315: 2415-2423.

3 Neutel CI, Johansen HL. Association between hypnotics use and increased mortality: causation or confounding? Eur J Clin Pharmacol 2015; 71: 637-642.

4 Ekstrom MP, Bornefalk-Hermansson A, Abernethy AP, et al. Safety of benzodiazepines and opioids in very severe respiratory disease: national prospective study. BMJ 2014; 348: g445.

5 Freemantle N, Marston L, Walters K, et al. Making inferences on treatment effects from real world data: propensity scores, confounding by indication, and other perils for the unwary in observational research. BMJ 2013; 347: f6409.

6 Currow DC, McDonald C, Oaten S, et al. Once-daily opioids for chronic dyspnea: a dose increment and pharmacovigilance study. J Pain Symp Manage 2011; 42: 388-399.

7 Rocker GM, Simpson AC, Young J, et al. Opioid therapy for refractory dyspnea in patients with advanced chronic obstructive pulmonary disease: patients' experiences and outcomes. CMAJ Open 2013; 1: E27-E36.

8 Abernethy AP, Currow DC, Frith P, et al. Randomised, double blind, placebo controlled crossover trial of sustained release morphine for the management of refractory dyspnoea. BMJ 2003; 327: 523-528.

9 Marciniuk DD, Goodridge D, Hernandez P, et al. Managing dyspnea in patients with advanced chronic obstructive pulmonary disease: a Canadian Thoracic Society clinical practice guideline. Can Respir J 2011; 18: 69-78.

Eur Respir J 2016; 48: 1520-1521 | DOI: 10.1183/13993003.01443-2016 | Copyright CERS 2016

\section{From the authors:}

We thank J. Downar and colleagues for their interest in our study [1] and their contributions to this important clinical issue. Downar and colleagues view our findings through the lens of palliative medicine. We recognise the role of opioids for symptom relief among individuals with chronic obstructive pulmonary disease (COPD) as part of end-of-life care. We intentionally excluded individuals who were receiving palliative care from our study, acknowledging that "goals of care and indications for opioid use may differ in this context" [1]. Our goal was instead to evaluate, from a respiratory safety perspective, the use of opioid drugs among the broader older adult COPD population. Our previous work on opioids in COPD [2] suggests that musculoskeletal pain is probably the main reason for opioid drug receipt in the older adult COPD population rather than respiratory symptoms. Opioids combined with non-opioid agents, like acetaminophen or aspirin, accounted for close to $90 \%$ of opioid use among older adults with COPD [2]. These combination opioid/non-opioid agents are unlikely to be used in end-of-life care and their use more likely reflects treatment of musculoskeletal pain, which commonly occurs in COPD [3, 4]. Opioids were also less commonly used among individuals with COPD with frequent exacerbations [2] and this group is more likely to be troubled by refractory respiratory symptoms. Less common use of opioids by these individuals supports the argument that opioids are prescribed for reasons other than palliation of respiratory symptoms.

Downar and colleagues suggest that our study findings may have been influenced by possible residual confounding by indication. While we acknowledged this possibility, we undertook several steps to minimise confounding. First, we excluded individuals receiving palliative care, since this group is near the end-of-life as a result of disease and opioids may be appropriately indicated in that setting. Second, our analyses were adjusted for a total of 33 different covariates, many of which are indicators of COPD severity, including respiratory exacerbation history, duration of $\mathrm{COPD}$, receipt of respiratory medications and presence of various comorbidities. We think it is unlikely that our results would be rendered nonsignificant if we additionally adjusted for one more covariate, dyspnoea symptoms, if this information were available to us. Third, we performed a sensitivity analysis examining for adverse respiratory outcomes associated with opioid use, distinguishing by COPD exacerbation history. COPD exacerbation history is the single best predictor of future exacerbation [5] and it is also associated with mortality [6]. We found increased all-cause mortality among incident opioid users, even in the healthiest subgroup with no exacerbations in the year prior to index [1]. Finally, we re-ran our analyses excluding individuals with lung malignancy and any malignancy, as these conditions might reflect palliative indications for opioid use. Significantly increased respiratory-related morbidity and mortality persisted even after excluding individuals with lung malignancy and any malignancy [1]. We feel that these steps help support our interpretation of the study findings. 